\title{
FILMES DE AMIDO E POLIETILENO: AVALIAÇÃO DA BIODEGRADAÇÃO POR VIA ENZIMÁTICA
}

\author{
Diego Márlon Ferro', Álvaro Vargas Júnior², Nei Fronza², Mara G. Novy Quadri*1 \\ ${ }^{1}$ Departamento de Engenharia Química e Engenharia de Alimentos, Universidade Federal de Santa \\ Catarina - UFSC \\ ${ }^{2}$ Instituto Federal Catarinense, Campus Concórdia - IFC \\ *e-mail: mara.quadri@ufsc.br
}

\begin{abstract}
RESUMO - Polímeros de petróleo têm sido extensivamente utilizados em diversos setores da industrialização em virtude de sua versatilidade, propriedades mecânicas e custo relativamente baixo. No entanto, trata-se de uma matéria-prima exaurível, que gera produtos poluidores. A característica da similaridade de filmes entre aqueles obtidos de fontes naturais em comparação aos de fonte fóssil traz à tona trabalhos com amido de milho, que parecem ser boa alternativa nesse quesito. Foram preparadas blendas poliméricas com polietileno linear de baixa densidade (PELBD) acrescido de cinco diferentes percentuais de amido. Após medidas de biodegradação enzimática pela enzima alfa-amilase durante 4 dias de tratamento, a maior perda de massa e resistência máxima à tração foram de 7,98\% e $14,78 \%$, respectivamente, na blenda com $50 \%$ de amido. A degradação é um fator fundamental que permite que as blendas se tornem possíveis substitutos de filmes plásticos provenientes de fonte fóssil, e seu estudo permite conhecer o potencial do uso da técnica de incorporação de amido como catalisador de degradação dos polímeros fósseis.
\end{abstract}

Palavras-chave: polímeros, biopolímeros, blendas, alfa-amilase, milho.

\section{INTRODUÇÃO}

Devido à demanda exacerbada por polímeros sintéticos e com aplicações nos mais variados setores da indústria, cresce a preocupação com os impactos ao meio ambiente. Essa não é uma preocupação recente, mas que ganha força com o passar do tempo. Além dos muitos benefícios que obtemos através do emprego de polímeros, deve-se considerar preocupação em propiciar um correto destino de depósito a ser dado após cumprido seu ciclo de utilização.

Na prática, ocorrem muitos problemas ambientais pois é sabido que o manejo correto e destino destes materiais, na maioria das vezes, não é feito. Dessa forma, tem-se um acúmulo crescente de depósito nocivo no meio ambiente, material este que por suas características intrínsecas demora centenas de anos para se decompor. Os plásticos sintéticos são considerados como inertes ao ataque microbiano devido à dificuldade de rompimento das cadeias poliméricas quando depositadas no meio ambiente; nestes casos respondem com um longo tempo de degradação (Rosa et al., 2001). 
A preocupação a nível mundial em relação aos impactos ambientais ocasionados pela intervenção humana se deve, em grande parte, à disseminação de polímeros biodegradáveis através da continuidade de pesquisas e desenvolvimentos de novos produtos (Rosa et al., 2001). Visando propiciar alternativas de matéria prima para o emprego em linhas fabris onde o plástico oriundo de fonte fóssil é muito utilizado, surge, como boa alternativa viável, o emprego de polímeros biodegradáveis. Quando comparados aos sintéticos, estes degradam rapidamente graças à influência dos diversos tipos de microrganismos presentes no meio em que o material será depositado. As fontes renováveis de materiais poliméricos oferecem uma parte da resposta na manutenção do desenvolvimento sustentável, considerando uma tecnologia econômica e ecologicamente atraente. As inovações no desenvolvimento de materiais biopoliméricos, a conservação de matérias primas fósseis, a degradabilidade biológica completa, a redução no volume de resíduos e compostabilidade, a redução do dióxido de carbono liberado, bem como um aumento de utilização de recursos agrícolas para a produção de novos materiais "verdes" são algumas das razões para o aumento do interesse mercadológico (Mohanty et al., 2005).

Seguindo esse raciocínio é que se volta a atenção ao amido, um dos mais conhecidos recursos renováveis capazes de propiciar a produção de cadeias poliméricas, os ditos plásticos biodegradáveis (Hocking, 1992; Mohanty et al., 2005). Dentre as opções existentes de biopolímeros, o amido ganha força devido ao seu preço, disponibilidade e habilidade como um termoplástico para produção de filmes biodegradáveis. Entretanto, os filmes obtidos exclusivamente desta matéria-prima são hidrófilos e por esse motivo sua degradabilidade ocorre devido ao enfraquecimento de propriedades mecânicas. Dessa forma, as blendas poliméricas utilizando termoplásticos naturais e polímeros sintéticos estão sendo estudados para melhorar o desempenho mecânico e reduzir a hidrofilicidade das misturas (Brandelero et al., 2012; Brandelero et al., 2011; Müller et al., 2012; Olivato et al., 2012; Olivato et al., 2012).

O enfraquecimento das propriedades mecânicas possui papel importante no auxílio da degradação de uma blenda polimérica, pois devido às forças de compressão, tensão e cisalhamento, o material será partido em porções menores, facilitando todo processo. Assim, os filmes termoplásticos podem sofrer estresse mecânico em condições de campo, auxiliando na degradação (Briassoulis et al., 2004; Briassoulis et al., 2005; Briassoulis et al., 2006). A aceleração na velocidade de degradação de termoplásticos oriundos de fontes naturais/renováveis é atribuída à atuação de diferentes vias tais como a enzimática, ataque de microrganismos, incidência de luz, dentre outros (Rosa; Filho, 2003).

Testes de bancada são efetuados para aferição do grau de degradação de misturas poliméricas. Segundo Rosa e Filho (2003), os testes de biodegradabilidade através de enzimas puras e isoladas, ao invés dos próprios microrganismos, servem como instrumento poderoso para a comparação rápida da degradação de polímeros de estruturas similares. Os efeitos da degradação são observados quando ocorre a perda das propriedades físicas do material. Este processo é geralmente caracterizado pela cisão das cadeias poliméricas, bem como pela quebra da estrutura no retículo cristalino.

O mecanismo de degradação por via enzimática pode ser resumido pela hidrólise de ligações peptídicas e outras que catalisam a hidrólise de ligações éster (Chandra; Rustgi, 1998). Este mecanismo é seguido pela oxidação biológica das cadeias poliméricas (catalisada 
pelas oxigenases), quebra das mesmas, gerando cadeias menores e bioassimilação destas pelos microrganismos. Este processo ocorre em polímeros contendo heterocadeias, tal como o amido (Scott, 2000).

Considerando o exposto acima, o presente trabalho desenvolveu filmes de blendas poliméricas amido-PE, e avaliou sua degradabilidade por via enzimática. A determinação do desempenho do filme é o objetivo do presente estudo, no qual se pretende obter filmes eficientes à função para a qual se destinam, sendo ao mesmo tempo menos danosos ao meio ambiente quando comparados aos filmes da cadeia petroquímica.

\section{MATERIAIS E MÉTODOS}

\subsection{Materiais}

Neste trabalho foi utilizado polietileno linear de baixa densidade (PELBD), fornecido pela Braskem Petroquímica SA (Triunfo/RS, Brasil), e amido de milho adquirido no comércio local. Glicerina, empregada como plastificante, foi acrescida de ácido cítrico, o qual atua como compatibilizante. $\mathrm{O}$ ácido cítrico é um ingrediente de grande interesse pois é um ácido orgânico que atende ao anseio maior do estudo, ou seja, a obtenção de filmes a partir de fontes renováveis. Enzima $(1,4)$ alfa-D-glicosídeo, declarada alfa-amilase em conformidade com as especificações de pureza recomendadas pelo Joint FAO/WHO Expert Committee on Food Additives (JECFA) e o Food Chemicals Codex (FCC), atuando em polissacarídeos amídicos, foi utilizada para avaliação da biodegradabilidade dos filmes.

\subsection{Preparação das Misturas e corpos-de-prova}

Material base consistindo de misturas de amido, glicerina e ácido cítrico, foi preparado e mantido em estufa a $72^{\circ} \mathrm{C}$ por $1 \mathrm{~h}$. Decorrido este tempo foram preparadas, além docontrole, as misturas contemplando cinco diferentes percentuais, variando de $10 \%$ à $50 \%$ de amido em polietileno, codificadas: F10, F20, F30, F40 e F50. Feito esse procedimento as misturas foram novamente colocadas em estufa $\left(72^{\circ} \mathrm{C} / 1 \mathrm{~h}\right)$.

Os filmes foram obtidos utilizando extrusora (Ax Plásticos, modelo LAB-16). Para a extrusão do material foi empregada velocidade de rosca de $36 \mathrm{rpm}$, e temperaturas nas zonas de aquecimento de $155^{\circ} \mathrm{C}$ nos dois primeiros estágios de contato e $160^{\circ} \mathrm{C}$ na terceira zona, onde o filme foi conformado. Para a laminação dos filmes foi empregada uma velocidade de $20 \mathrm{rpm}$ nos roletes.

\subsection{Ensaios de biodegradação}

A avaliação da biodegradabilidade foi realizada segundo as normas ASTM D6003, em triplicata de cada filme. Corpos de prova preparados seguindo as especificações da norma ASTM D882-01, foram devidamente pesados e deixados em estufa por 48 horas, sendo novamente pesados antes de serem colocados na presença da enzima. Os dispositivos de biodegradação foram preparados contendo $20 \mathrm{~mL}$ solução tampão fosfato $\mathrm{pH}$ 7,0 e adição da enzima à $1 \%$ do volume.Os ensaios foram efetuados em shaker, com tempo de permanência 
de 96 horas a $37^{\circ} \mathrm{C}$ e agitação orbital de $110 \mathrm{rpm}$. Ao final do ensaio, as amostras degradadas foram cuidadosamente lavadas e secas em estufa, respeitando as mesmas condições atribuídas no momento da primeira pesagem.

\section{RESULTADOS E DISCUSSÃO}

As blendas com adição de amido, uma vez postas em tratamento enzimático por alfaamilase, propiciam facilidade ao rompimento da integridade das mesmas. Isso é observado quando do aumento do percentual de amido nos tratamentos, aumentando também a perda de força de tração, que na avaliação chega ao valor máximo de 14,78\% em F50, como demonstra a série de linha pontilhada, que possui comportamento aproximado à série paralela de linha contínua, referente à perda de massa, no gráfico 1 .

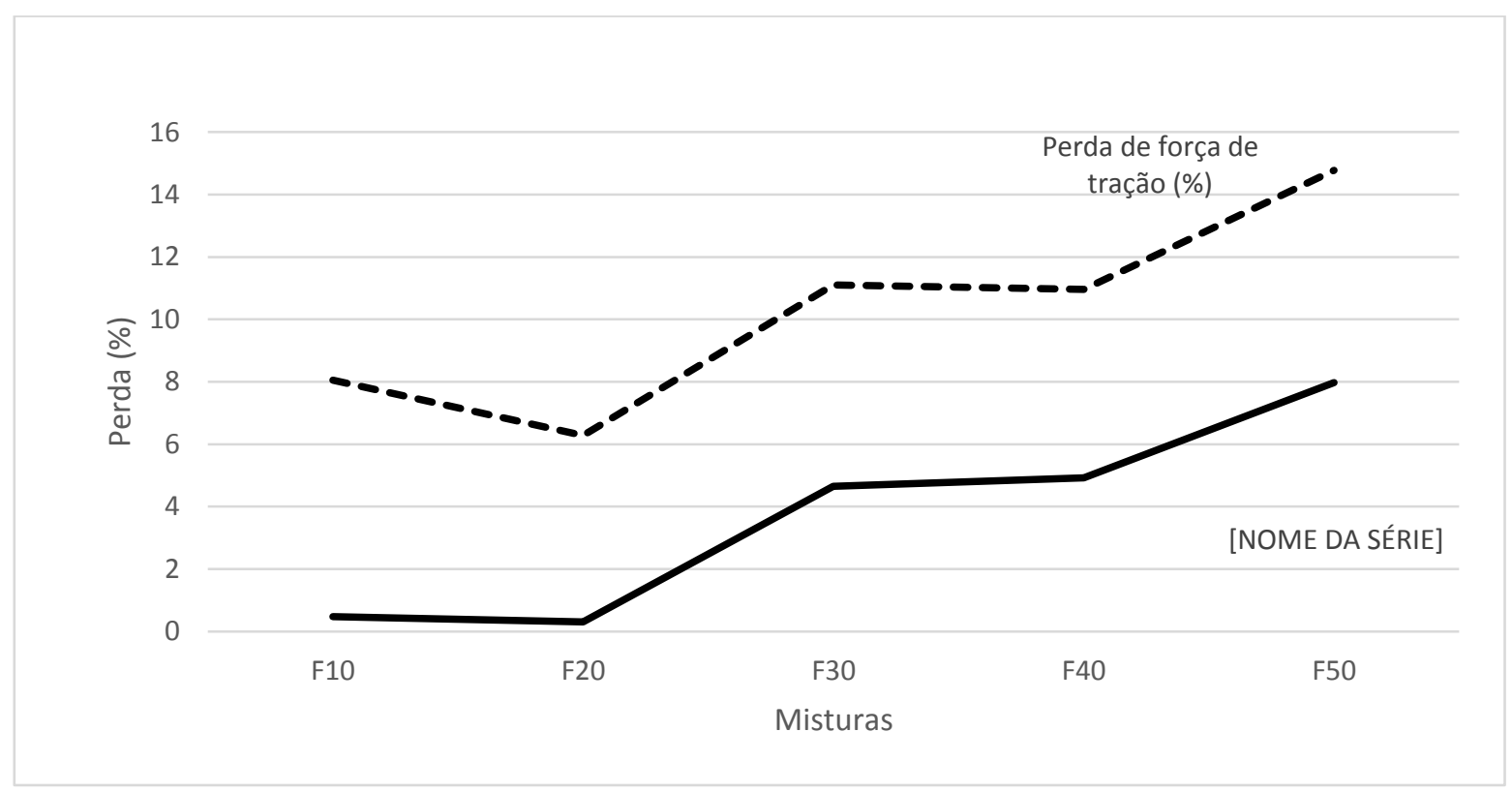

Gráfico 1: Perdas percentuais das amostras em ensaios de tração e aferição mássica.

Segundo Rabello (2000), cargas adicionadas às blendas podem contribuir a propagação de trincas, resultado da diminuição à resistência ao impacto, proveniente do aumento da viscosidade do material fundido pelas cargas inertes, o que também responde por dificuldades no processamento do material.

A degradação é dependente de vários propriedades químicas e físicas. Severini et al., (1987) relata que em tratamentos de 10.000 horas de exposição, em blendas com polietileno a cristalinidade deste permanece estável. Segundo Koller et al., (1996) e Koenig et al., (1995), quando da adição de amido no composto, este leva à redução das propriedades mecânicas devido à interface matriz/carga com baixa compatibilidade e a não ocorrência do mecanismo de molhamento (as camadas de água existentes na superfície do amido não permitem a remoção do ar presente na interface). $\mathrm{O}$ amido age como um concentrador de tensão no composto, induzindo as trincas durante o ensaio de tração e diminuindo a resistência à tração resultante (Iovino et al., 2008). 
A formação de blendas visando maior proximidade às propriedades dos polímeros convencionais, traz à tona uma das maiores dificuldades: a obtenção de boas propriedades mecânicas e apelo ambiental, o que significa que deve haver, na estrutura do filme, um aumento da adesão interfacial entre o amido e o material sintético. Para tanto faz-se uso de plastificantes e compatibilizantes como o ácido cítrico, o qual é adicionado para promover as reações de esterificação e de reticulação na interface entre as cadeias poliméricas. Isto, de acordo com Zhang e Sun (2004), tem sido eficaz para o controle da morfologia de vários sistemas poliméricos.

Da mesma forma como é importante propiciar boas características mecânicas aos filmes produzidos, é também fundamental que estes atendam aos anseios de um material biodegradável. Para tanto, o teste de degradação por via enzimática foi estabelecido como método de aferição do potencial catalisador que o amido exerce numa blenda, de modo a facilitar a quebra da integridade física da estrutura matricial do polímero. Encerrado o tratamento enzimático, é possível observar o acúmulo de amido nos dispositivos de degradação (Figura 1).
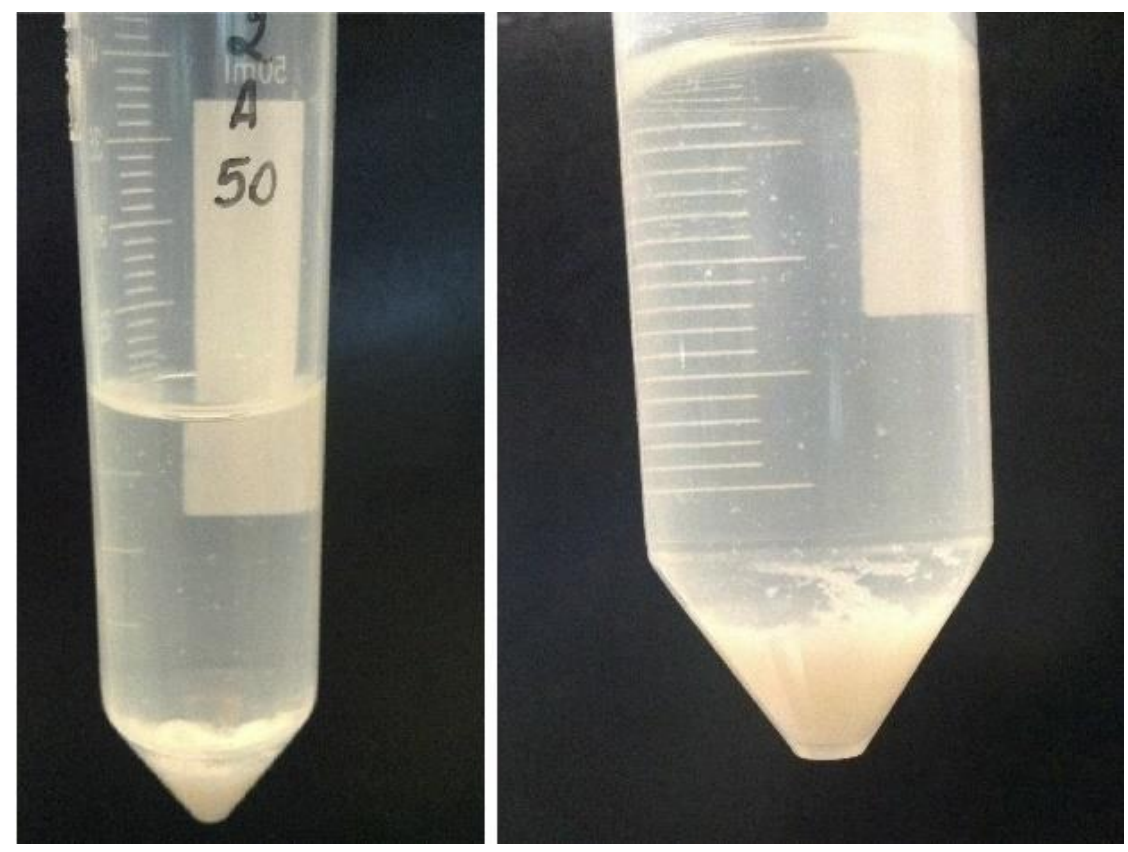

Figura 1 - Dispositivo de tratamento enzimático permite visualização do amido extraído após concluído ensaio com amostra de $50 \%$ de amido em sua composição.

Um decréscimo na perda de massa é observado entre as amostras F10 e F20, correspondendo a uma menor perda de força à tração da mistura F20; atribui-se a esta mistura a melhor relação entre resistência e potencial degradativo. Os demais filmes mostram perdas de massa maiores que $4,5 \%$, com consequente perda de força à tração maiores que $10 \%$. A maior perda de massa é observada na mistura onde há a maior quantidade de amido na composição, chegando ao valor próximo de $8 \%$, resultado encontrado no estudo de Rosa et al. (2005) em tratamento de $144 \mathrm{~h}$. A perda de massa promovida pelo tratamento enzimático, e consequente perda de resistência à tração dos filmes feitos de blendas contendo de 10 a 50\% de amido é apresentada na Tabela 1. 
Tabela 1: Perda de massa e da Resistência máxima a tração das blendas (F10-F50) em relação ao filme controle (FC) após biodegradação enzimática.

\begin{tabular}{ccc}
\hline Filme & Perda de massa (\%) & Perda de força de tração $(\%)$ \\
\hline FC & - & - \\
F10 & 0,48 & 8,05 \\
F20 & 0,31 & 6,28 \\
F30 & 4,65 & 11,10 \\
F40 & 4,92 & 10,96 \\
F50 & 7,98 & 14,78 \\
\hline
\end{tabular}

(-) manteve as características, sem alteração.

Esses resultados estão intimamente associados à acessibilidade do amido das misturas à enzima alfa-amilase, a qual se encontra diretamente envolvida na degradação de biomateriais a base de amido (Azevedo et al., 2003). A perda de massa dos filmes após o tratamento por via enzimática, torna-os porosos pela perda do amido, de modo a representar facilidade ao ataque por microrganismos e incorporação de oxigênio, por exemplo, aumentando assim a degradação do polímero por vias biológicas e oxidativas. $\mathrm{O}$ amido residual no filme continua a contribuir na degradação em razão de possuir moléculas com dois grupos funcionais importantes: o grupo -OH que é suscetível a reações de substituição e o C-O-C e que é suscetível a quebra da cadeia (Huang et al., 1990).

\section{CONSIDERAÇÕES FINAIS}

No presente estudo foi possível observar a efetividade do tratamento enzimático como técnica de medida de biodegradabilidade de blendas poliméricas. Um percentual satisfatório de perda de massa, e em conformidade com estudos encontrados em literatura, pode ser observado, em menor tempo de tratamento para misturas com igual percentual de amido na composição. Também permitiu visualizar que o filme contendo $20 \%$ de amido é a mistura que fornece a melhor propriedade mecânica quando comparado com filme convencional.

\section{REFERÊNCIAS BIBLIOGRÁFICAS}

ASTM (2001). Standard test method for tensile properties of thin plastic sheeting. Designation D882. In: Annual Book of American Standard Testing Methods (edited by ASTM). Pp. 162-170. Philadelphia, PA: American Society for Testing and Materials.

ASTM D6003-96 - "Standard method for determining weight loss from plastic materials exposed to simulated municipal solid-wast (MSM) aerobic compost environment". Philadelphia, 1996.

AZEVEDO, H. S.; GAMA, F. M.; REIS, R. L. In Vitro Assessment of the Enzymatic Degradation of Several Starch Based Biomaterials. Biomacromol., v. 4, n. 6, p. 17031712, 2003. 
BRANDELERO, R. P. H.; GROSSMANN, M. V.; YAMASHITA, F. Films of starch and poly(butylene adipate co-terephthalate) added of soybean oil (SO) and Tween 80. Carb. Polym., v. 90, n. 4, p. 1452-1460, 2012.

BRANDELERO, R. P. H.; GROSSMANN, M. V. E.; YAMASHITA, F. Effect of the method of production of the blends on mechanical and structural properties of biodegradable starch films produced by blown extrusion. Carb. Polym., v. 86, n. 3, p. 1344-1350, 2011 .

BRIASSOULIS, D. Mechanical Design Requirements for Low Tunnel Biodegradable and Conventional Films. Biosyst. Eng., v. 87, n. 2, p. 209-223, 2004.

BRIASSOULIS, D. The effects of tensile stress and the agrochemical Vapam on the ageing of low density polyethylene (LDPE) agricultural films. Part I. Mechanical behaviour. Polym. Deg. Stab., v. 88, n. 3, p. 489-503, 2005.

BRIASSOULIS, D. Mechanical behaviour of biodegradable agricultural films under real field conditions. Polym. Deg. Stab., v. 91, n. 6, p. 1256-1272, 2006.

CHANDRA, R.; RUSTGI, R. Biodegradable polymers. Progr. in Polym. Sci., v. 23, n. 7, p. 1273-1335, 1998.

HOCKING, P. J. The Classification, Preparation, and Utility of Degradable Polymers. J. of Macrom. Sci., Part C, v. 32, n. 1, p. 35-54, 1992.

HUANG, J.-C.; SHETTY, A. S.; WANG, M.-S. Biodegradable plastics: A review. Adv. in Polym. Technol., v. 10, n. 1, p. 23-30, 1990.

IOVINO, R. et al. Biodegradation of poly(lactic acid)/starch/coir biocomposites under controlled composting conditions. Polym. Deg. Stab., v. 93, n. 1, p. 147-157, 2008.

KOENIG, M. F.; HUANG, S. J. Biodegradable blends and composites of polycaprolactone and starch derivatives. Polym., v. 36, n. 9, p. 1877-1882, 1995.

KOLLER, I.; OWEN, A. J. Starch-Filled PHB and PHB/HV copolymer. Polym. Intern., v. 39, n. 3, p. 175-181, 1996.

MOHANTY, A. K.; MISRA, M.; DRZAL, L. T. Nat. Fibers, Biopolym. and Biocomp., Taylor \& Francis, 2005.

MÜLlER, C. M. O.; LAURINDO, J. B.; YAMASHITA, F. Composites of thermoplastic starch and nanoclays produced by extrusion and thermopressing. Carbohyd. Polym., v. 89 , n. 2, p. 504-510, 2012.

OLIVATO, J. B. et al. Effect of organic acids as additives on the performance of thermoplastic starch/polyester blown films. Carbohyd. Polym., v. 90, n. 1, p. 159-164, 2012.

OLIVATO, J. B. et al. Citric acid and maleic anhydride as compatibilizers in starch/poly(butylene adipate-co-terephthalate) blends by one-step reactive extrusion. Carbohyd. Polym., v. 87, n. 4, p. 2614-2618, 2012.

RABELLO, M. Aditiv. de polim., Artliber, 2000. 
ROSA, D. S.; FILHO, R. P. Biodegradação: um ensaio com polímeros. Moara, 2003.

ROSA, D. S.; FRANCO, B. L. M.; CALIL, M. R. Biodegradabilidade e propriedades mecânicas de novas misturas poliméricas. Polím., 11: 82-88 p. 2001.

ROSA, D. S.; LOPES, D. R.; CALIL, M. R. Thermal properties and enzymatic degradation of blends of poly(E-caprolactone) with starches. Polym., v. 24, n. 6, p. 756-761, 2005.

SCOTT, G. 'Green' polymers. Polym. Deg. Stab., v. 68, n. 1, p. 1-7, 2000.

SEVERINI, F., GALLO, R., and IPSALE, S. "Environmental degradation of stabilized LDPE. Later stages." Polym. Deg. Stab., v. 17, p. 57-64, 1987.

ZHANG, J.-F.; SUN, X. Mechanical Properties of Poly(lactic acid)/Starch Composites Compatibilized by Maleic Anhydride. Biomacromol., v. 5, n. 4, p. 1446-1451, 2004. 